\title{
Effect of Coriolis force on Rayleigh-Bénard convection with internal heat generation
}

\author{
Noor Arshika $\mathbf{S}^{1 *}$, Mohamed El Hadramy Oumar ${ }^{1}$ and S Pranesh ${ }^{2}$ \\ Post Graduate Student, Department of Mathematics, Christ (Deemed to be University), Bengaluru ${ }^{1}$ \\ Professor, Department of Mathematics, Christ (Deemed to be University), Bengaluru ${ }^{2}$
}

Received: 10-March-2019; Revised: 22-May-2019; Accepted: 25-May-2019

(C)2019 Noor Arshika S et al. This is an open access article distributed under the Creative Commons Attribution (CC BY) License, which permits unrestricted use, distribution, and reproduction in any medium, provided the original work is properly cited.

\begin{abstract}
The objective of this paper is to analyze the influence of the Coriolis force and internal heat source on Rayleigh-Bénard convection in a Boussinesquian fluid of depth $d$. A linear theory which is oriented towards the normal mode analysis technique is used for this mono-diffusive convection in order to find the criteria for the onset of Rayleigh-Bénard convection. The eigenvalue of the said problem was obtained by the use of the Galerkin method in the cases of rigid-rigid, rigid-free, and free-free velocity boundary combinations considering the isothermal and adiabatic temperature boundaries that determine the stability of the system. The effects of various parameters, Taylor number and the internal Rayleigh number are put under consideration only for stationary convection. Treating Taylor number as a critical parameter, shown that it plays a major role in stabilization of the system in case of any particular infinitesimal disturbance. The destabilization of the system has been possible with rotation by treating internal Rayleigh numbers as a critical parameter since the increase in values of the internal Rayleigh number advances the onset of convection. Oscillatory convection seems highly improbable as the scaled frequency of oscillation remains less than 0 for all combinations of Prandtl number, internal Rayleigh number, and Taylor number.
\end{abstract}

\section{Keywords}

Thermal convection, Rotation, Internal heat source, Stability, Boussinesquian fluid, Rayleigh number.

\section{Introduction}

The effect of internal heat generation has a significant role in various applications including fire and in the combustion studies as well as storage of the radioactive materials and many others. It is found that in many situations, the material tends to offer its own source of heat, leading to an alternate manner by which a convective flow can happen by means of the local heat generation within the layer. Such a scenario takes place through radioactive decay or the exothermic reaction as well as the nuclear reaction which can happen to occur within the material. Due to this internal heat generation, we can find the thermal gradient inside and outside of the earth's crust, which is soaked with multi-segment fluids, which enables the convective flow, and therefore transforming the thermal energy towards the earth's surface.

\footnotetext{
*Author for correspondence
}

Hence, there is an immense significance of internal heat generation turns out to be very apparent in various applications that includes storage of radioactive materials, geophysics, reactor safety analysis. The movement of any fluid element can be distinguished by their unique features which are translation, rate of deformation and the rotation taking place in the space. When we consider the rotation of fluid with some velocity, we see that the pressure rapidly increases in such a way that the pressure is independent of the direction of the angular velocity generated by the rotating fluid. Yekasi et al. [1] studied the role of internal heat sources on RayleighBénard convection driven by suction-injection combination under $1 \mathrm{~g}$ and $\mu \mathrm{g}$ situations between an upper free and a lower rigid boundary showing that internal heat source results in stabilization of the system upon infinitesimal disturbance on it. Haajizadeh et al. [2] made a study on the time independent natural convection that takes place within a rectangular porous media with a uniform internal heat as well as cooling from the side wall. The study 
was made in two cases, low Rayleigh numbers and large Rayleigh numbers, it was found out that in the core region, the heat that is generated is carried upward by a vertical convection wherein the lateral conduction can be neglected whereas in the boundary layers the heat is removed by lateral cooling. The analytical study when the natural convection is taking place subjected to volumetrically internal heat generation with the effect of the magnetic field was studied by Benos and Sarris [3], wherein the method of asymptotic expansion has been used to obtain the analytical solution that is valid for the cavities with the comparatively larger ratio when considering significantly low Rayleigh numbers. Malashetty and Heera [4] made a study on the effect of rotation and anisotropy on the onset of a double diffusive convection in a porous layer and found that the existence of unstable motions in case of rotating porous medium does not depend on a specific range of Prandtl number as opposed to the case of viscous fluid flow. Riahi [5] studied the inertial and rotational effects on an oscillatory flow in a horizontal layer and the study showed that the Coriolis effect is able to enhance the physical domain for an oscillatory flow as opposed to the inertial force. Another study made by Deepika et al. [6] shows that there are potential regions of sub-critical instabilities for increasing values of internal heat source parameter $\mathrm{Q}$ in the problem of thermal convection in a horizontal fluidsaturated porous layer where the flow is governed by the Brinkman extension of Darcy's law. The onset of convection with a parabolic basic temperature profile was first studied by Roberts [7] in the study of convection in a horizontal layer with internal heat generation. Bhadauria et al. [8] studied the effect of internal heating and time-periodic gravity modulation on Rayleigh-Bénard convection in a vertically oscillating micropolar fluid. It was found that in a convective system, the effect of internal Rayleigh number has a destabilizing effect. Riahi [9] studied the linear flow instability in oscillatory and stationary modes of disturbance in a horizontal porous layer at the time of alloy solidification and found that they are both stabilizing and destabilizing effects of the Coriolis force on the flow in a porous layer. When there is some kind of obstacle which has been uniformly heated in a Newtonian fluid that satisfies the Boussinesquian approximation, then the stagnation point flow of the fluid and the pattern of the micro-rotation becomes almost similar when compared to the stagnation point flow when orthogonal which was a study done by Borrelli et al. [10]. Combining the effects of rotation and with the internal heating in a fluid layer which is viscosity dependent was a study made by Bhadauria and Kiran [11], and the results shows internal heating destabilizes the system. Thermal convection taking place in a rotating anisotropy porous medium was a study carried out by Vanishree and Siddheshwar [12], by applying the linear stability analysis on the convection where temperature-dependent viscosity is considered. The oscillatory and stationary convection were both considered separately and the oscillatory convection seems highly improbable. The natural convection which is taking place in a rotating anisotropy porous medium with an internal heat generation was a study made by Bhadauria et al. [13], wherein the linear theory is performed using the normal mode analysis technique and the corresponding stability analysis derives the conditions for the stationary as well as convection which is oscillatory. The effect of rotation in a horizontal anisotropic porous layer was also studied by Malashetty and Swamy [14], in which the linear theory is carried out by using the normal mode analysis method and the nonlinear theory is based on the truncated Fourier series analysis. The normal mode analysis technique shows that the linear theory provides the criteria for the onset of stationary as well as the oscillatory convection. A direct transition to turbulence in a rotating Bénard convection was studied by Niemala and Donnelly [15] in which a transition from a certain heat transfer by conduction implied directly to a turbulent convective flow in a rotating Bénard cell is observed. The effect of rotation on Rayleigh-Bénard convection was studied extensively by Professor Chandrasekhar [16] and added in the recent developments by Knobloch [17] wherein plenty of results have been derived that has been extensively used in the study of the paper. Peter Vadasz [18] also studied the natural convection in the rotating flows wherein the effect of rotation plays an important role in the pure fluids and also in the porous media. The paper mainly concentrates on the comparison of fluid layers with and without the effect of rotation. Ramachandramurthy et al. [19] studied the temperature dependent volumetric heat source on the onset of the Rayleigh-Bénard convection. It was found that a linear stability analysis is used for the stationary convection and the Taylor number delays the heat transfer.

\section{List of symbols}

$l, m$ - Horizontal wave numbers

$d$-Distance between the two plates

$\hat{k}$ - Unit vector in the vertical direction

$a$ - Wave number 
$p$ - Hydrodynamic pressure

$\vec{q}-(U, V, W)$ Velocity

$\operatorname{Pr}=\frac{\mu}{\rho_{R} k}-$ Prandtl number

$R_{a}=\frac{\rho_{R} \alpha g \Delta T d^{3}}{\mu k}-$ Rayleigh number

$t$ - Time

$T$ - Temperature

$T a=\frac{4 d^{4} \Omega_{o}^{2} \rho_{R}^{2}}{\mu^{2}}$ - Taylor number

$R_{i}=\frac{Q d^{2}}{k}-$ Internal Raleigh number

$U, V, W$ - Dimensional horizontal and vertical velocity components

$\vec{g}$ - Acceleration due to gravity

$k$ - Thermal conductivity

$x$ - Horizontal coordinate

$z$ - Vertical coordinate

$\alpha$ - Coefficient of thermal expansion

$\Omega$ - Angular velocity of rotation

$\rho$ - Density

$\rho_{R}$ - Density of the fluid taken at reference temperature

$T=T_{m}$

$\Delta T$ - Temperature gradient

$\sigma$ - Growth rate of perturbation

$\nabla-\frac{\partial}{\partial x} \hat{i}+\frac{\partial}{\partial y} \hat{j}+\frac{\partial}{\partial z} \hat{k}$ Vector differential operator

$\nabla^{2}-\frac{\partial^{2}}{\partial x^{2}}+\frac{\partial^{2}}{\partial y^{2}}+\frac{\partial^{2}}{\partial z^{2}} \quad$ Three-dimensional Laplacian

operator

$\nabla_{1}^{2}-\frac{\partial^{2}}{\partial x^{2}}+\frac{\partial^{2}}{\partial y^{2}}$ Two-dimensional Laplacian operator

$\mu$ - Dynamic viscosity of the fluid

$\xi-z$-component of vorticity $\left(\frac{\partial v}{\partial x}-\frac{\partial u}{\partial y}\right)$

$\omega$ - Scaled frequency of oscillation

Subscripts:

$c$ - Critical value

$b$ - Basic state

Superscripts:

* - Dimensionless quantity

- Perturbed quantity

$O$ - Oscillatory

$S$ - Stationary

\section{Mathematical formulation}

A Boussinesquian fluid in an infinite rotating horizontal layer having depth $d$ in the presence of internal heat source is considered. Also considering $(x, y, z)$ as a cartesian coordinate system where its origin is taken at the lower boundary while the z-axis is pointing vertically upwards. $\Delta \mathrm{T}$ is taken as the difference of temperature between the lower plate and the upper plate. This infinite horizontal layer is considered to be rotating uniformly with a constant angular velocity $\Omega_{0}$ around z-axis (Figure 1).

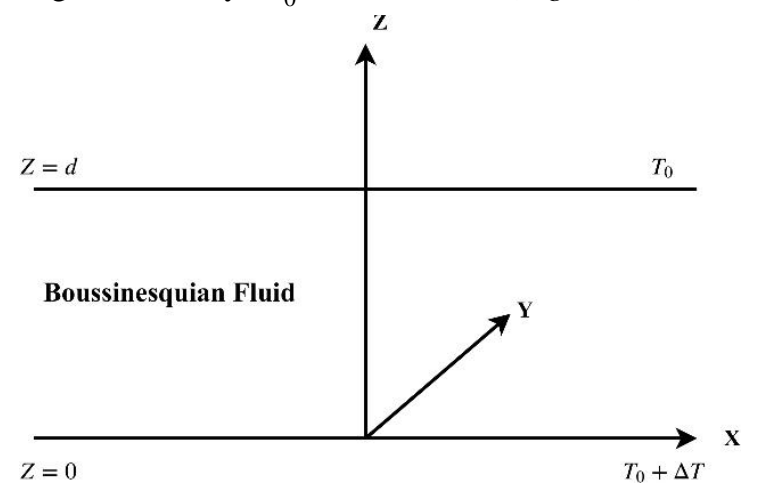

Figure 1 Physical configuration for the RayleighBénard situation in Boussinesquian fluid

The applicable governing equations to describe the Boussinesquian flow under the considered model are:

\section{Continuity Equation:}

$$
\nabla \cdot \vec{q}=0 \text {, }
$$

Conservation of linear Momentum:

$$
\left.\begin{array}{r}
\rho_{o}\left[\frac{\partial \vec{q}}{\partial t}+(\vec{q} \cdot \nabla) \vec{q}\right]=-\nabla p+\rho \vec{g}+2 \rho_{R}(\vec{q} \times \vec{\Omega}) \\
+\mu \nabla^{2} \vec{q}
\end{array}\right\},
$$

Conservation of Energy:

$$
\frac{\partial T}{\partial t}+(\vec{q} \cdot \nabla) T=k \nabla^{2} T+Q\left(T-T_{o}\right),
$$

Equation of state:

$$
\rho=\rho_{R}\left[1-\alpha\left(T-T_{o}\right)\right] .
$$

\section{Basic state}

The fluid is at rest in this state and heat is transferred only by conduction. The fluid in this state is described by:

$$
p=p_{b}(z), \rho=\rho_{b}(z), T=T_{b}(z), \vec{q}_{b}=0 .
$$

Substituting Equation 5 into Equation 2-4, we get:

$$
\frac{d p_{b}}{d z}=-\rho_{b} g \hat{k},
$$


Noor Arshika S et al.

$$
\begin{aligned}
& k \frac{d^{2}\left(T_{b}-T_{o}\right)}{d z}+Q\left(T_{b}-T_{o}\right)=0, \\
& \rho_{b}=\rho_{R}\left[1-\alpha\left(T_{b}-T_{o}\right)\right] .
\end{aligned}
$$

Solving the differential Equation 7, we get

$$
T_{b}=T_{o}+\Delta T \frac{\operatorname{Sin}\left(\sqrt{R_{i}}\left(1-\frac{z}{d}\right)\right)}{\operatorname{Sin}\left(\sqrt{R_{i}}\right)} .
$$

\section{Linear stability analysis}

Let the system be perturbed from the basic state. Equations in this state are given by,

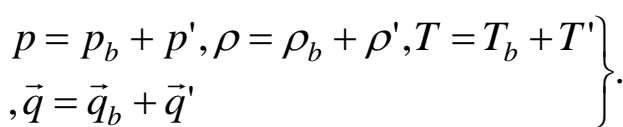

The prime quantities indicate that they are infinitesimal perturbations while subscript 'b' implies the value in the basic state. Equation 10 is substituted into Equation 1-4, also making use of the basic state of Equation 5-9, we get,

$$
\begin{aligned}
& \left.\begin{array}{l}
\nabla \cdot \vec{q}^{\prime}=0, \\
\rho_{R}\left[\frac{\partial \vec{q}^{\prime}}{\partial t}+\left(\vec{q}^{\prime} \cdot \nabla\right) \vec{q}^{\prime}\right]=-\nabla p^{\prime}+\rho_{R} \alpha T^{\prime} g \hat{k}^{\prime}+\mu \nabla^{2} \vec{q} \\
+2 \rho_{R}\left(\vec{q}^{\prime} \times \Omega\right)
\end{array}\right\}, \\
& \left.\begin{array}{rl}
\frac{\partial T^{\prime}}{\partial t}+\left(\vec{q}^{\prime} \cdot \nabla\right) T^{\prime}=-\omega^{\prime} \frac{d T_{b}}{d z} & +k \nabla^{2} T^{\prime} \\
& +Q T^{\prime}
\end{array}\right\}, \\
& \rho^{\prime}=-\rho_{R} \alpha T^{\prime} .
\end{aligned}
$$

Substituting Equation 14 in 12 and nondimensionalizing resultant equation and Equation 13 using the definitions as follows:

$$
\left.\begin{array}{l}
\left(x^{*}, y^{*}, z^{*}\right)=\frac{1}{d}(x, y, z), T^{*}=\frac{T^{\prime}}{\Delta T}, \\
t^{*}=\frac{t}{\left(\frac{d^{2}}{k}\right)}, \omega^{*}=\frac{\omega^{\prime}}{\left(\frac{d^{2}}{k}\right)}, p^{*}=\frac{p}{p_{1}}
\end{array}\right\} .
$$

Using Equation 15 in Equation 12 and 13, and dropping the asterisks for simplicity we get the resultant equations as:

$$
\left.\begin{array}{rl}
\frac{1}{\operatorname{Pr}}\left[\frac{\partial \vec{q}}{\partial t}+(\vec{q} \cdot \nabla) \vec{q}\right] & =-P_{0} \nabla p \\
& +R_{a} T \hat{k}+\frac{d^{2} 2 \rho_{R}(\vec{q} \times \vec{\Omega})}{\mu}+\nabla^{2} \vec{q}
\end{array}\right\},
$$

$\frac{\partial T}{\partial t}+(\vec{q} \cdot \nabla) T=W f(z)+\nabla^{2} T+R_{i} T$,

where $f(z)=\frac{\sqrt{R_{i}} \operatorname{Cos}\left(\sqrt{R_{i}}(1-z)\right)}{\operatorname{Sin}\left(\sqrt{R_{i}}\right)}$.

Neglecting the non-linear terms in Equation 16 and 17 , we get

$$
\left.\begin{array}{rl}
\frac{1}{\operatorname{Pr}}\left[\frac{\partial \vec{q}}{\partial t}\right]=-P_{0} \nabla p & +R_{a} T \hat{k} \\
& +\frac{d^{2} 2 \rho_{R}(\vec{q} \times \vec{\Omega})}{\mu}+\nabla^{2} \vec{q}
\end{array}\right\},
$$

Applying curl twice on Equation 18 to eliminate the term containing pressure, we get,

$$
\left.\begin{array}{r}
\frac{1}{\operatorname{Pr}}\left[\frac{\partial\left(\nabla^{2} W\right)}{\partial t}\right]=-R_{a} T \hat{k} \\
+\frac{2 d^{2} \rho_{R} \Omega_{0}}{\mu} \frac{\partial \xi}{\partial z}+\nabla^{4} W
\end{array}\right\} .
$$

Applying curl once on Equation 18, we get,

$$
\frac{1}{\operatorname{Pr}}\left[\frac{\partial \xi}{\partial t}\right]=\frac{2 d^{2} \rho_{R} \Omega_{0}}{\mu} \frac{\partial W}{\partial z}+\nabla^{2} \xi .
$$

Equation 21 is called the vorticity transport equation. The infinitesimal perturbation $\mathrm{W}, \$ \mathrm{xi} \$$ and $\mathrm{T}$ are assumed to be periodic waves and therefore they have a normal mode solution which is in the form

$$
\left[\begin{array}{c}
W \\
\xi \\
T
\end{array}\right]=\left[\begin{array}{l}
W(z) \cdot \exp (\sigma t+i l x+i m y) \\
\xi(z) \cdot \exp (\sigma t+i l x+i m y) \\
T(z) \cdot \exp (\sigma t+i l x+i m y)
\end{array}\right]
$$

Equation (22) is substituted into equations (19)-(21), we get

$$
\left.\begin{array}{r}
\frac{\sigma}{\operatorname{Pr}}\left[\left(D^{2}-a^{2}\right) W\right]=-R_{a} a^{2} T \\
+\frac{d^{2}}{\mu} \rho_{R}\left[\Omega_{0} D \xi\right]+\left(D^{2}-a^{2}\right)^{2} W
\end{array}\right\},
$$




$$
\begin{aligned}
& \sigma T=W f(z)+\left(D^{2}-a^{2}\right)^{2} T+R_{i} T, \\
& \frac{\sigma}{\operatorname{Pr}} \xi=\left(D^{2}-a^{2}\right) \xi+\frac{d^{2}}{\mu} \rho_{R} \Omega_{0} D W .
\end{aligned}
$$

where $D=\frac{d}{d z}$. We discuss marginal stability by putting both stationary and oscillatory convection under consideration.

\section{Galerkin method}

Equations 23-25 are solved using the Galerkin method. We choose trial functions such that they satisfy the given boundary conditions however they need not satisfy the differential equations thereby obtaining an approximate solution of the differential equation. This will lead to residuals upon substituting these trial functions into the differential equations. This method demands the residuals to be orthogonal to each of the trial functions. In this method, we thereby expand the velocity and the temperature as follows:

$W(z, t)=\Sigma A_{j} W_{j}(z)$,

$\xi(z, t)=\Sigma B_{j} \xi_{j}(z)$

$T(z, t)=\Sigma C_{j} T_{j}(z)$.

Where $W_{j}(z), \xi_{j}(z)$, and $T_{j}(z)$ are the trial functions discussed and $A_{j}, B_{j}$, and $C_{j}$ are constants.

Multiplying the Equations 23-25 with $W, T$, and $\xi$ respectively and integrating them between 0 and 1 with respect to $\mathrm{z}$ and substituting $W, T$, and $\xi$ in the following manner, $W=A W_{1}, \quad \xi=B \xi_{1}$, $T=C T_{1}$ in which the constants are $A, B, C$ with $W_{1}, T_{1}, \xi_{1}$ being the trial functions satisfying the boundary conditions

$$
\begin{aligned}
& \frac{\sigma}{\operatorname{Pr}}\left\langle W_{1}\left(D^{2}-a^{2}\right) W_{1}\right\rangle=R_{a} a^{2}\left\langle W_{1} T_{1}\right\rangle \\
& \left.+\frac{d^{2}}{\mu} \rho_{R} \Omega_{0}\left\langle W_{1} D \xi_{1}\right\rangle+\left\langle W_{1}\left(D^{2}-a^{2}\right)^{2} W_{1}\right\rangle\right\}, \\
& \sigma\left\langle T_{1}^{2}\right\rangle=\left\langle T_{1} W_{1} f(z)\right\rangle+\left\langle T_{1}\left(D^{2}-a^{2}\right)^{2} T_{1}\right\rangle+R_{i}\left\langle T_{1}^{2}\right\rangle, \\
& \frac{\sigma}{\operatorname{Pr}}\left\langle\xi_{1}^{2}\right\rangle=\left\langle\xi_{1}\left(D^{2}-a^{2}\right) \xi_{1}\right\rangle+\frac{d^{2}}{\mu} \rho_{R} \Omega_{0}\left\langle\xi_{1} D W_{1}\right\rangle .
\end{aligned}
$$

$\langle\cdots\rangle$ in Equation (37) denotes integration with respect to $\mathrm{z}$ from 0 to 1 . This procedure yields the following equation for Rayleigh number $R_{a}$ :

$$
R_{a}=\frac{\frac{\sigma}{\operatorname{Pr}}\left(G_{2}\right)-\left(\frac{T a E_{1} E_{2}}{\frac{\sigma}{\operatorname{Pr}}\left(F_{3}\right)-G_{3}}\right)-F_{4}}{\frac{a^{2}\left(E_{3}\right)}{\sigma\left(F_{1}\right)-G_{1}-R_{i}\left(F_{1}\right)}} .
$$

where,

$$
\begin{aligned}
& F_{1}=\left\langle T_{1}^{2}\right\rangle, \\
& F_{2}=\left\langle W_{1}^{2}\right\rangle, \\
& F_{3}=\left\langle\xi_{1}^{2}\right\rangle, \\
& F_{4}=\left\langle W_{1}\left(D^{2}-a^{2}\right)^{2} W_{1}\right\rangle, \\
& G_{1}=\left\langle T_{1}\left(D^{2}-a^{2}\right) T_{1}\right\rangle, \\
& G_{2}=\left\langle W_{1}\left(D^{2}-a^{2}\right) W_{1}\right\rangle, \\
& G_{3}=\left\langle\xi_{1}\left(D^{2}-a^{2}\right) \xi_{1}\right\rangle, \\
& E_{1}=\left\langle\xi_{1} D W_{1}\right\rangle, \\
& E_{2}=\left\langle W_{1} D \xi_{1}\right\rangle, \\
& E_{3}=\left\langle T_{1} W_{1} f(z)\right\rangle\left\langle W_{1} T_{1}\right\rangle .
\end{aligned}
$$

Setting $\sigma=0$ for stationary convection, the equation (29) becomes

$$
R_{a}^{s}=\frac{\left(G_{1}+R_{i} F_{1}\right)\left(T a E_{1} E_{2}+G_{3} F_{4}\right)}{-a^{2} E_{3} G_{3}} .
$$

Setting $\sigma=i \omega$ for oscillatory convection, the equation (29) becomes

$$
\left.R_{a}^{o}=\frac{\begin{array}{c}
\omega^{2} Y_{1} Y_{3} Y_{6}+Y_{1} Y_{5} Y_{6}-\omega^{2} Y_{2} Y_{4} Y_{6}+\omega^{2} Y_{1} Y_{4} Y_{7} \\
+\omega^{4} Y_{2} Y_{3} Y_{7}+\omega^{2} Y_{2} Y_{5} Y_{7}+i \omega L
\end{array}}{Y_{6}^{2}+\omega^{2} Y_{7}^{2}}\right\} .
$$

Where,

$$
\begin{aligned}
& \left.\begin{array}{r}
L=Y_{1} Y_{4} Y_{6}+\omega^{2} Y_{2} Y_{3} Y_{6}+Y_{2} Y_{5} Y_{6}-\omega^{2} Y_{1} Y_{3} Y_{7} \\
-Y_{2} Y_{5} Y_{7}+\omega^{2} Y_{2} Y_{4} Y_{7}
\end{array}\right\}, \\
& Y_{1}=-\left(G_{1}+R_{i} F_{1}\right), \\
& Y_{2}=F_{1} \text {, } \\
& Y_{3}=-\frac{1}{\operatorname{Pr}^{2}} G_{2} F_{3} \text {, }
\end{aligned}
$$


Noor Arshika S et al.

$$
\begin{aligned}
& Y_{4}=-\frac{1}{\operatorname{Pr}}\left(G_{2} G_{3}+F_{3} F_{4}\right), \\
& Y_{5}=-T a E_{1} E_{2}+G_{3} F_{4}, \\
& Y_{6}=-a^{2} E_{3} G_{3}, \\
& Y_{7}=\frac{1}{\operatorname{Pr}} a^{2} E_{3} F_{3} .
\end{aligned}
$$

Since $R_{a}$ is not a complex number, we conclude that $\omega=0$ or $L=0$.

The condition $L=0$ can be used to obtain the frequency of oscillations $\omega$ and thereby obtaining the Rayleigh number in oscillatory convection as follows:

$$
\begin{aligned}
& \omega^{2}=\frac{Y_{1} Y_{5} Y_{7}-Y_{2} Y_{5} Y_{6}-Y_{1} Y_{4} Y_{6}}{Y_{2} Y_{3} Y_{6}-Y_{1} Y_{3} Y_{7}+Y_{2} Y_{4} Y_{7}}, \\
& \omega^{2} Y_{1} Y_{3} Y_{6}+Y_{1} Y_{5} Y_{6}-\omega^{2} Y_{2} Y_{4} Y_{6}+\omega^{2} Y_{1} Y_{4} Y_{7} \\
& R_{a}^{o}=\frac{+\omega^{4} Y_{2} Y_{3} Y_{7}+\omega^{2} Y_{2} Y_{5} Y_{7}}{Y_{6}^{2}+\omega^{2} Y_{7}^{2}} .
\end{aligned}
$$

The boundary combinations considered in this paper are:

Rigid-Rigid isothermal.

$\mathrm{W}=\mathrm{DW}=\mathrm{D} \xi=\mathrm{T}=0$, at $\mathrm{z}=0,1$.

Rigid-Free isothermal.

$\mathrm{W}=\mathrm{DW}=\mathrm{D} \xi=\mathrm{T}=0$, at $\mathrm{z}=0$,

$\mathrm{W}=\mathrm{D}^{2} \mathrm{~W}=\mathrm{D} \xi=\mathrm{T}=0$, at $\mathrm{z}=1$.

Free-Free isothermal.

$\mathrm{W}=\mathrm{D}^{2} \mathrm{~W}=\mathrm{D} \xi=\mathrm{T}=0$, at $\mathrm{z}=0,1$.

Rigid-Rigid adiabatic.

$\mathrm{W}=\mathrm{DW}=\mathrm{D} \xi=\mathrm{DT}=0$, at $\mathrm{z}=0,1$.

Rigid-Free adiabatic.

$\mathrm{W}=\mathrm{DW}=\mathrm{D} \xi=\mathrm{DT}=0$, at $\mathrm{z}=0$,

$\mathrm{W}=\mathrm{D}^{2} \mathrm{~W}=\mathrm{D} \xi=\mathrm{DT}=0$, at $\mathrm{z}=1$.

Free-Free adiabatic.

$\mathrm{W}=\mathrm{D}^{2} \mathrm{~W}=\mathrm{D} \xi=\mathrm{DT}=0$, at $\mathrm{z}=0,1$.

Table 1 The trial functions that satisfy the various boundary conditions

\begin{tabular}{llll}
\hline Trial functions & Rigid-Rigid & Rigid-Free & Free-free \\
\hline $\mathrm{W}_{\mathbf{1}}$ & $z\left(1-2 z^{2}+z^{3}\right)$ & $z^{4}-\frac{5}{2} z^{3}+\frac{3}{2} z^{2}$ & $z\left(1-2 z^{2}+z^{3}\right)$ \\
\hline$\xi_{1}$ & $\operatorname{Cos} \pi z$ & $\operatorname{Cos} \pi z$ & $\operatorname{Cos} \pi z$ \\
\hline Isothermal temperature $\mathrm{T}_{1}$ & $z-z^{2}$ & $z-z^{2}$ & $z-z^{2}$ \\
\hline Adiabatic temperature $\mathrm{T}_{1}$ & 1 & 1 & 1 \\
\hline
\end{tabular}

\section{Results}

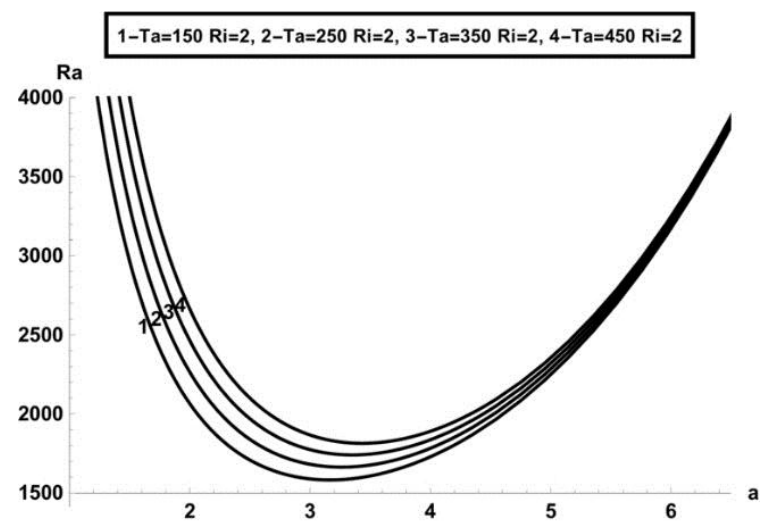

Figure 2 against for various values of with in case of rigid-rigid isothermal

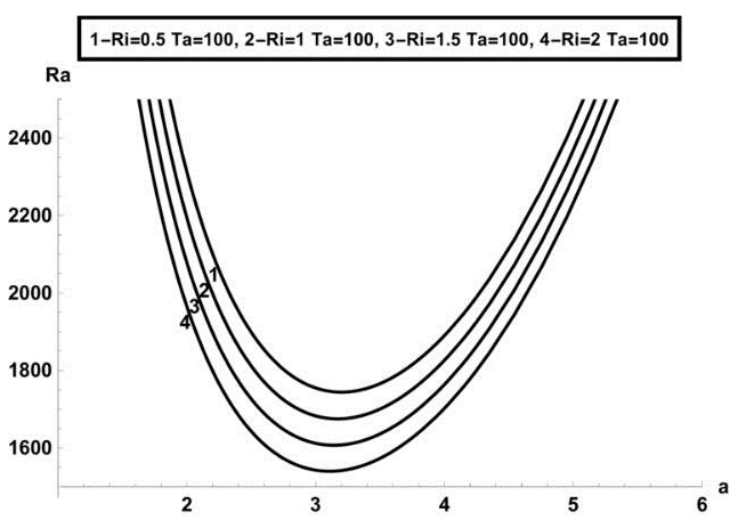

Figure $3 R_{a}$ against $a$ for various positive values of $R_{i}$ with $T a=100$ in case of rigidrigid isothermal 
International Journal of Advanced Technology and Engineering Exploration, Vol 6(54)

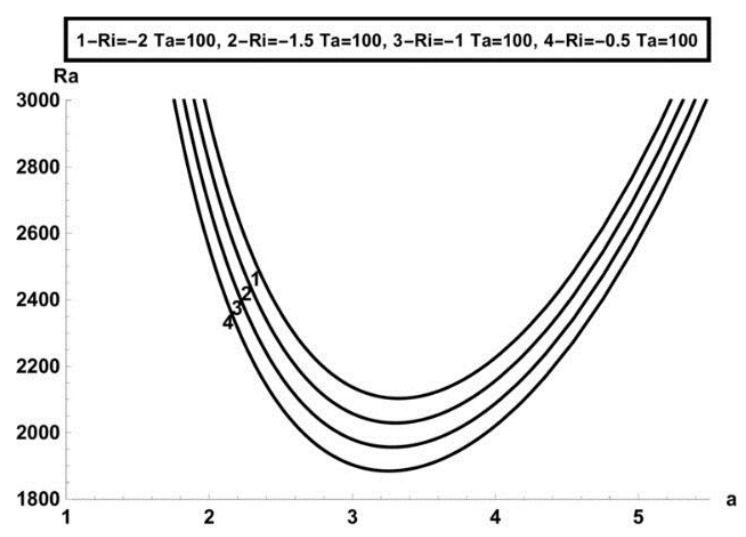

Figure $4 R_{a}$ against $a$ for various negative values of $R_{i}$ with $T a=100$ in case of rigidrigid isothermal

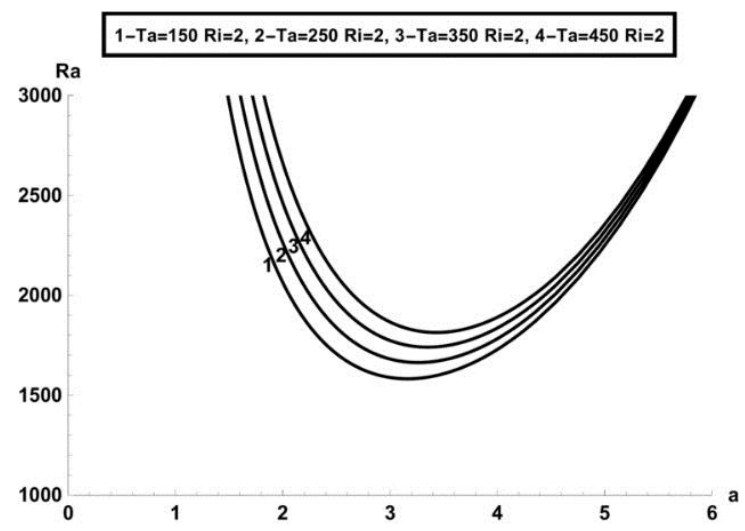

Figure $5 R_{a}$ against $a$ for various values of $\mathrm{Ta}$ with $R_{i}=2$ in case of rigid-free isothermal

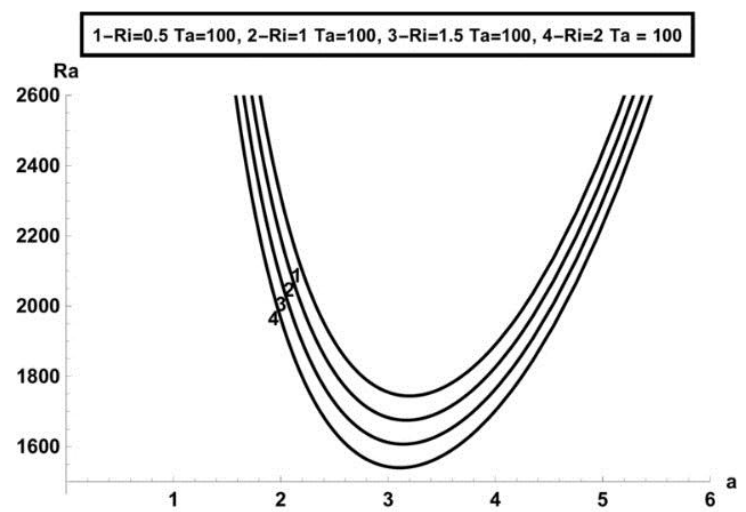

Figure $6 R_{a}$ against $a$ for various positive values of $R_{i}$ with $T a=100$ in case of rigidfree isothermal

139

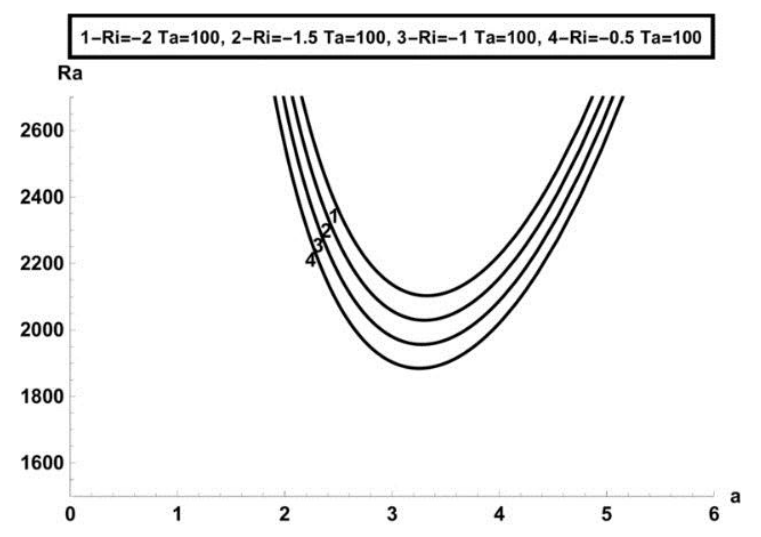

Figure $7 R_{a}$ against $a$ for various negative values of $R_{i}$ with $T a=100$ in case of rigidfree isothermal

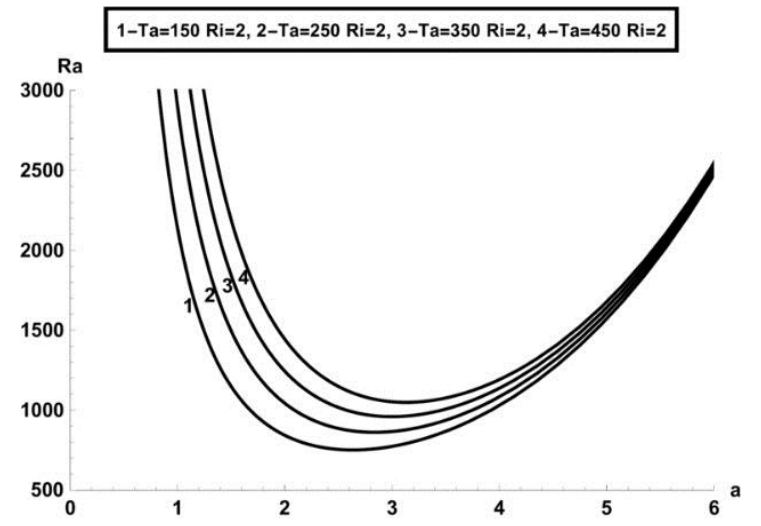

Figure $8 R_{a}$ against $a$ for various values of Ta with $R_{i}=2$ in case of free-free isothermal

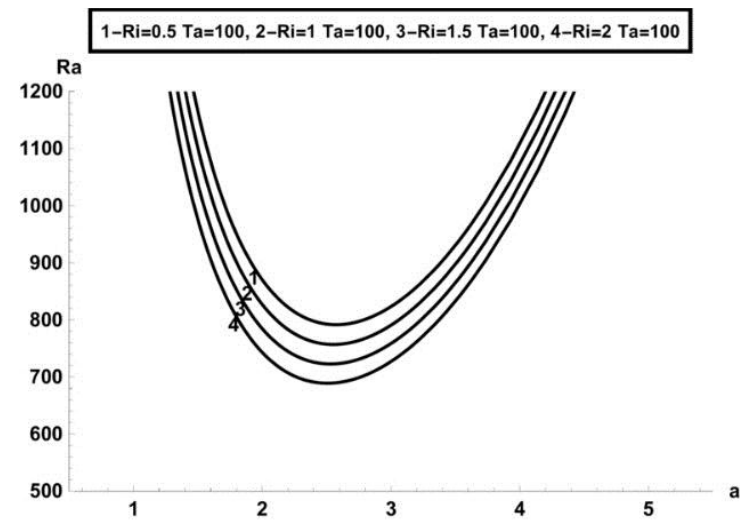

Figure $9 R_{a}$ against $a$ for various positive values of $R_{i}$ with $T a=100$ in case of freefree isothermal 
Noor Arshika S et al.

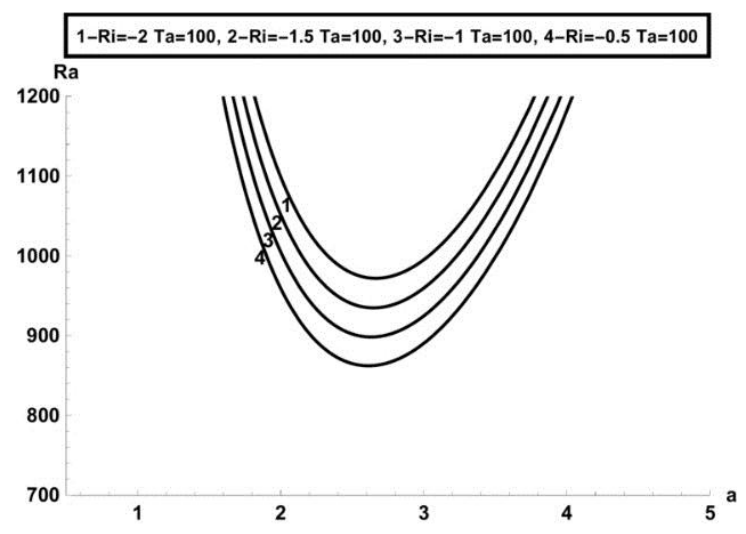

Figure $10 R_{a}$ against $a$ for various negative values of $R_{i}$ with $T a=100$ in case of freefree isothermal

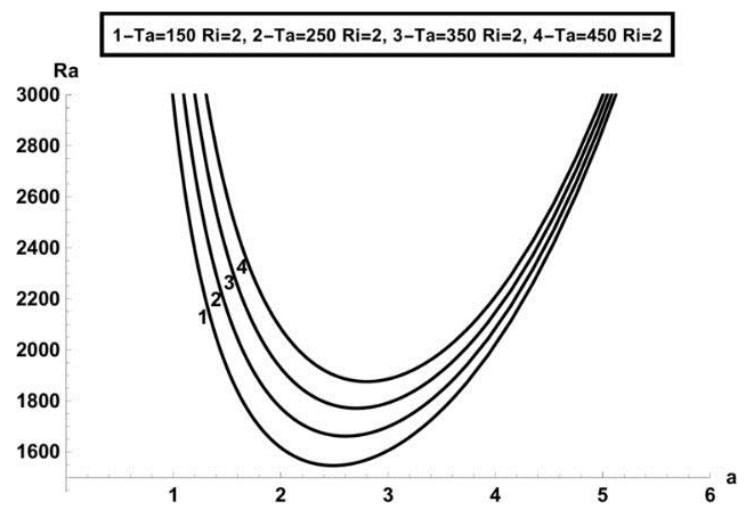

Figure $11 R_{a}$ against $a$ for various values of $T a$ with $R_{i}=2$ in case of rigid-rigid adiabatic.

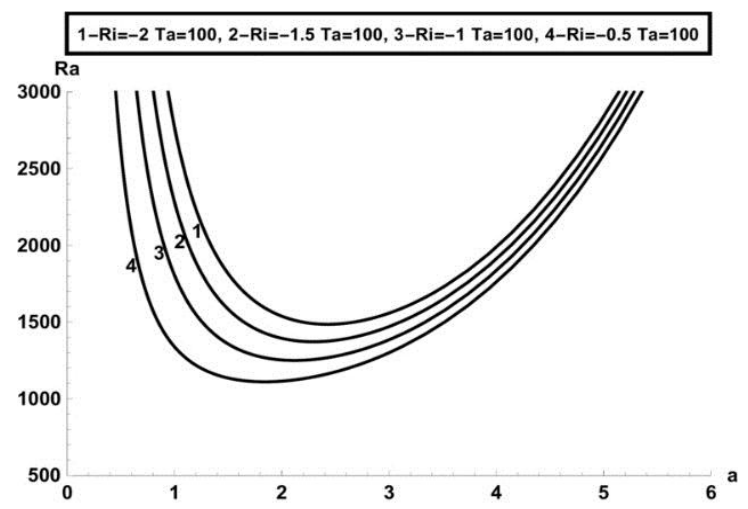

Figure $12 R_{a}$ against $a$ for various negative values of $R_{i}$ with $T a=100$ in case of rigidrigid adiabatic

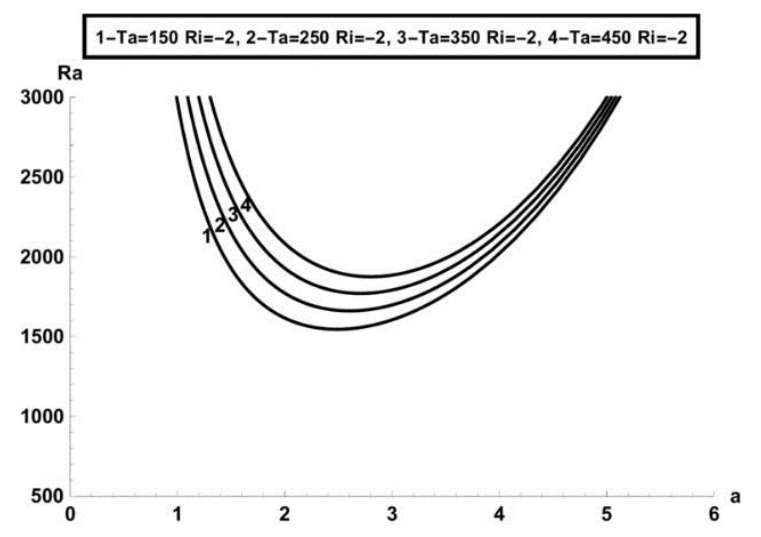

Figure $13 R_{a}$ against $a$ for various values of $T a$ with $R_{i}=2$ in case of rigid-free adiabatic.

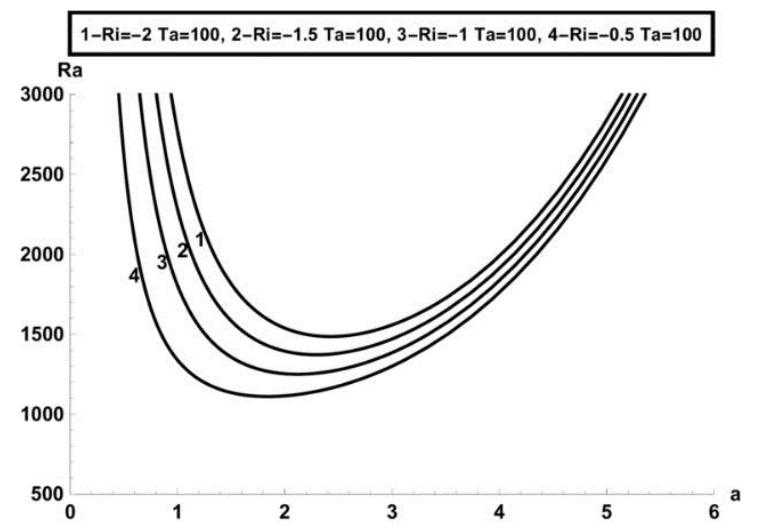

Figure $14 R_{a}$ against $a$ for various negative values of $R_{i}$ with $T a=100$ in case of rigidfree adiabatic

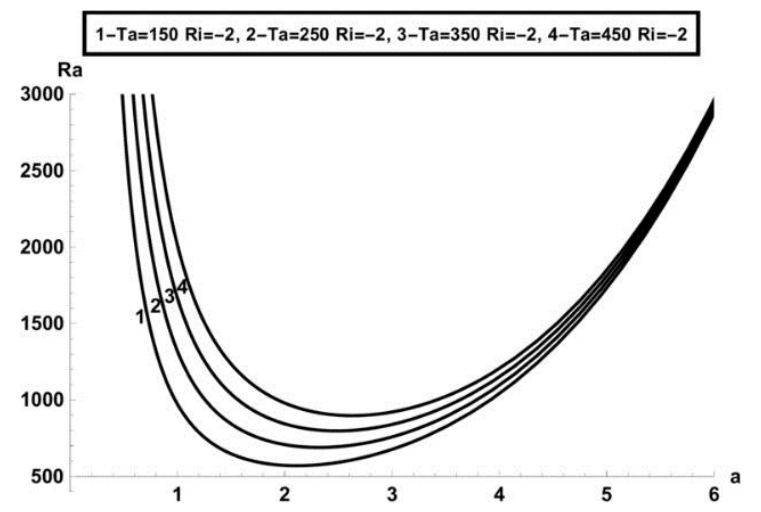

Figure $15 R_{a}$ against $a$ for various values of $T a$ with $R_{i}=2$ in case of free-free adiabatic 


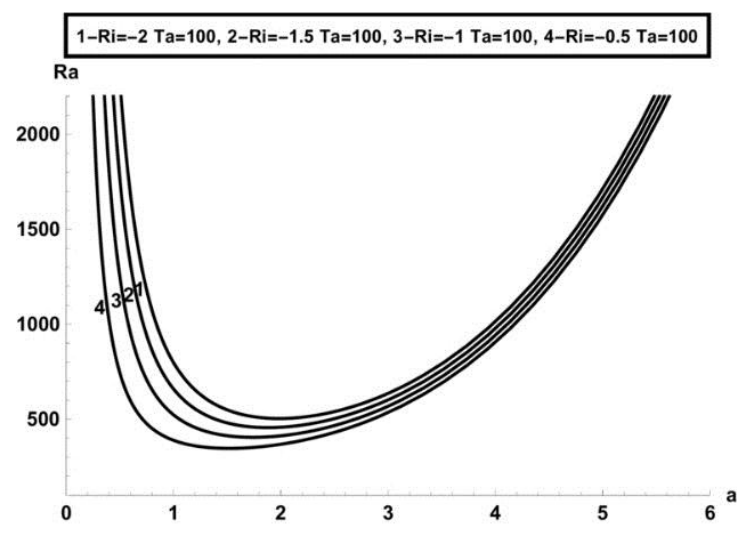

Figure $16 R_{a}$ against $a$ for various negative values of $R_{i}$ with $T a=100$ in case of freefree adiabatic

\section{Discussion}

The rotation's effect on the onset Rayleigh-Bénard convection in the presence internal heat generation in Boussinesquian fluid using linear stability analysis was investigated in this paper. The main emphasis of this study is to investigate the above effects on the onset of convection in the stationary mode since oscillatory convection appears to be highly improbable. The Eigen value of the problem was obtained with the help of Galerkin technique as a function of $T a$ and $R_{i}$. The linear stability was used to express the condition of stability in terms of the critical Rayleigh number $R_{a_{c}}$. Figure 2 is the plot of $R_{a}$ against $a$ for various values of $T a$ in case of rigid-rigid isothermal boundary conditions. It was found that the increase in the Taylor number $T a$ results in the increase in $R_{a}$, thereby delaying the onset of convection. Therefore, the onset of convection gets delayed by the decrease in the heat transport due to the increase of $T a$. Thus, rotation strongly stabilizes the system.

Figures 3 and 4 are the plot of $R_{a}$ against $a$ for various positive and negative values of $R_{i}$ in case of rigid-rigid isothermal boundary conditions. We found that the increase in values of $R_{i}$ results in the decrease of $R_{a}$, thereby advancing the onset of convection. Therefore, the onset of convection is advanced by the increase in heat supply due to the increase of $R_{i}$. Thus, increasing internal Rayleigh number $R_{i}$ results in the destabilization of the system.
Figures 5, 6, 7 are the plot of $R_{a}$ against $a$ for various values of $T a$, positive and negative internal Rayleigh number respectively in case of rigid-free isothermal boundary conditions. Figures 8,9,10 are the plot of $R_{a}$ against $a$ for various values of $T a$, positive and negative internal Rayleigh number respectively in case of rigid-free isothermal boundary conditions. Figures 11 and 12 are the plot of $R_{a}$ against $a$ for various values of $T a$, and negative internal Rayleigh number respectively in case of rigidrigid adiabatic boundary conditions. Figures 13 and 14 are the plot of $R_{a}$ against $a$ for various values of $T a$, and negative internal Rayleigh number respectively in case of rigid-free adiabatic boundary conditions. Figures 15 and 16 are the plot of $R_{a}$ against $a$ for various values of $T a$, and negative internal Rayleigh number respectively with respect to free-free adiabatic boundary conditions. The plots for isothermal and adiabatic temperature boundary conditions are qualitatively similar. Equation (33) can be used to conclude that oscillatory convection is highly improbable since $\omega^{2}$ remains less than 0 for all combinations of the parameters $\operatorname{Pr}, R_{i}$ and $T a$. Thus, we conclude that the oscillatory motions are not possible for this problem.

\section{Conclusion and future work}

The stability of a rotating infinite horizontal layer of a Boussinesquian fluid in the presence of internal heat generation is investigated. Linear stability analysis was made using normal mode analysis and Galerkin technique. The conclusions drawn from the study are:Both internal Rayleigh number and electric Rayleigh number destabilize the system.

(i)The role of Taylor number $T a$ is to strongly stabilize the system while the internal Rayleigh number destabilizes the system.

(ii) $R_{c}^{R R}>R_{c}^{R F}>R_{c}^{F F}, R_{c}$ is the critical Rayleigh number and the subscripts represents the different velocity boundary conditions. This holds for both adiabatic and isothermal boundaries.

(iii)In the case of a rotating horizontal layer of Boussinesquian fluid, stationary convection seems to be the mode of instability preferred rather than oscillatory convection.

A further analysis can be made for the following.

- The effect of internal heat generation and rotation on the onset of Rayleigh-Benard electro convection in Boussinesquian fluid. 
- The effect of internal heat generation and rotation on the onset of Rayleigh-Benard magneto convection in Boussinesquian fluid.

\section{Acknowledgment}

Authors would like to thank CHRIST (Deemed to be University) for giving an opportunity to work on this project.

\section{Conflicts of interest}

The authors have no conflicts of interest to declare.

\section{References}

[1] Yekasi V, Pranesh S, Bathul S. Effects of suctioninjection combination and internal heat source in a fluid with internal angular momentum under $1 \mathrm{~g}$ and $\mu \mathrm{g}$. Journal of Computer and Mathematical Sciences. 2018; 9(6):526-37.

[2] Haajizadeh M, Ozguc AF, Tien CL. Natural convection in a vertical porous enclosure with internal heat generation. International Journal of Heat and Mass Transfer. 1984; 27(10):1893-902.

[3] Benos L, Sarris IE. Analytical study of the magnetohydrodynamic natural convection of a nanofluid filled horizontal shallow cavity with internal heat generation. International Journal of Heat and Mass Transfer. 2019; 130:862-73.

[4] Malashetty MS, Heera R. The effect of rotation on the onset of double diffusive convection in a horizontal anisotropic porous layer. Transport in Porous Media. 2008.

[5] Riahi DN. Inertial and coriolis effects on oscillatory flow in a horizontal dendrite layer. Transport in Porous Media. 2007; 69(3):301-12.

[6] Deepika N, Narayana PA, Hill AA. Onset of darcybrinkman convection with a uniform internal heat source and vertical throughflow. International Journal of Thermal Sciences. 2017; 117:136-44.

[7] Roberts PH. Convection in horizontal layers with internal heat generation. Theory. Journal of Fluid Mechanics. 1967; 30(1):33-49.

[8] Bhadauria BS, Hashim I, Siddheshwar PG. Effect of internal-heating on weakly non-linear stability analysis of Rayleigh-Bénard convection under g-jitter. International Journal of Non-Linear Mechanics. 2013; $54: 35-42$

[9] Riahi DN. On stationary and oscillatory modes of flow instability in a rotating porous layer during alloy solidification. Journal of Porous Media. 2003; 6(3).

[10] Borrelli A, Giantesio G, Patria MC. Effect of temperature on the MHD stagnation-point flow past an isothermal plate for a Boussinesquian Newtonian and micropolar fluid. International Journal of Numerical Methods for Heat \& Fluid Flow. 2018; 28(6):1315-34.

[11] Bhadauria BS, Kiran P. Effect of rotational speed modulation on heat transport in a fluid layer with temperature dependent viscosity and internal heat source. Ain Shams Engineering Journal. 2014; 5(4):1287-97.
[12] Vanishree RK, Siddheshwar PG. Effect of rotation on thermal convection in an anisotropic porous medium with temperature-dependent viscosity. Transport in Porous Media. 2010.

[13] Bhadauria BS, Kumar A, Kumar J, Sacheti NC, Chandran P. Natural convection in a rotating anisotropic porous layer with internal heat generation. Transport in Porous Media. 2011.

[14] Malashetty MS, Swamy M. The effect of rotation on the onset of convection in a horizontal anisotropic porous layer. International Journal of Thermal Sciences. 2007; 46(10):1023-32.

[15] Niemela JJ, Donnelly RJ. Direct transition to turbulence in rotating Bénard convection. Physical Review Letters. 1986.

[16] Chandrasekhar S. Hydrodynamic and hydromagnetic stability. Courier Corporation; 2013.

[17] Knobloch E. Rotating convection: recent developments. International Journal of Engineering Science. 1998; 36(12-14):1421-50.

[18] Vadasz P. Natural convection in rotating flows. Handbook of Thermal Science and Engineering. 2018.

[19] Ramachandramurthy V, Uma D, Kavitha N. Effect of non-inertial acceleration on heat transport by rayleighbénard magnetoconvection in boussinesq-stokes suspension with variable heat source. International Journal of Applied Engineering Research. 2019; 14(9):2126-33.

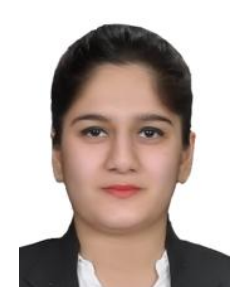

Noor Arshika is doing her Masters of Science in mathematics at CHRIST (Deemed to be University). Her research interests lie in the field of Fluid Mechanics.

Email: noor.arshika@maths.christuniversity.in

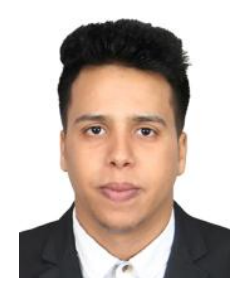

Mohamed El Hadramy Oumar is doing his Masters of Science in mathematics at CHRIST (Deemed to be University). His research interests lie in the field of Fluid Mechanics.

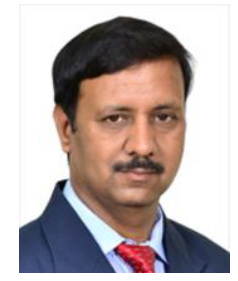

Dr. Pranesh $\mathbf{S}$ completed his Ph.D from Bangalore University. $\mathrm{He}$ is currently working as a Professor in the Department of Mathematics, CHRIST (Deemed to be University). His research interests lie in the field of Fluid Mechanics. 